\title{
Efecto del peso al sacrificio sobre la calidad de la canal y de la carne de corderos Corriedale puros y cruza
}

\author{
Effect of live weight at slaughter on carcass and meat quality in pure \\ Corriedale and crossbred lambs \\ G Bianchi ${ }^{* 1}$, G Garibotto ${ }^{1}$, O. Feed ${ }^{2}$, O Bentancur ${ }^{1}$ y J Franco ${ }^{1}$ \\ ${ }^{1}$ Universidad de la República, Facultad de Agronomía, Estación Experimental "Dr. Mario A. Cassinoni” \\ (EEMAC), Departamento de Producción Animal y Pasturas, Unidad Calidad de Producto, Uruguay \\ ${ }^{2}$ Universidad de la República, Facultad de Veterinaria, Estación Experimental “Dr. Mario A. Cassinoni” (EEMAC), Uruguay.
}

\section{SUMMARY}

The effects of slaughter weights on light lambs ( $\mathrm{n}=45): 22.3 \pm 2.8 \mathrm{~kg}$ and $60 \pm 2.4$ days of age and heavy lambs $(\mathrm{n}=21): 43.1 \pm$ $6.3 \mathrm{~kg}$ and $169 \pm 8.9$ days of age on carcass and meat quality of 24 females, 23 cripthorchid and 19 castrated Corriedale $(n=21)$ and Hampshire Down x Corriedale $(\mathrm{n}=23)$ and Southdown $x$ Corriedale $(\mathrm{n}=22)$ lambs was studied. Slaughter weights affected $(\mathrm{P} \leq 0.05)$ all carcass characteristics evaluated. Heavy lambs had better conformation $(0.320 \mathrm{vs} 0.190 \mathrm{~kg} / \mathrm{cm}, 1.09 \mathrm{vs} 1.07,55.7$ vs 51.9 carcass and leg compacticity of heavy and light lambs, respectively), better fat grade (15.7 vs $7.3 \mathrm{~mm}$ GR, heavy and light lambs, respectively) and more favourable tissue composition ( $2.9 \mathrm{vs} 1.9$ meat/bone, $2.5 \mathrm{vs} 2.2$ meat / fat, heavy and light lambs, respectively). Nevertheless, light lamb carcasses had a better commercial yield of high value cuts than those of heavy lambs. Interms of the measurable quality characteristics of meat, only the $\mathrm{pH}$ was affected by slaughter weight. In the sensory analysis, sex and cross only affected meat tenderness. Meat from females or castrated lambs was more tender than cripthorchid lambs, particularly if they were crossbred lambs. Slaughter weights affected all sensory characteristics, with the meat from heavy lambs receiving the best scores in terms of tenderness, flavor quality and acceptability.

Palabras clave: cordero, canal, carne, calidad.

key words: lambs, carcass, meat, quality.

\section{INTRODUCCION}

En Uruguay la producción de carne de cordero ha crecido en forma sostenida en los últimos años (Salgado 2003), existiendo dos modalidades de producción predominantes: cordero liviano $(22-24 \mathrm{~kg}$ de peso vivo al sacrificio) y cordero pesado ( $>34 \mathrm{~kg}$ de peso vivo $\mathrm{y} \geq 3,5$ de estado corporal al sacrificio). Las categorías sacrificadas a nivel nacional son mayormente machos castrados, provenientes de genotipos puros laneros o doble propósito, aunque en los últimos años la práctica de criptorquidea inducida (Hudson y col 1968) y el uso de cruzamientos terminales han cobrado importancia en la producción de corderos pesados. En el ámbito internacional, el efecto de estos factores productivos sobre la calidad de la canal y sobre todo de la carne de cordero han sido ampliamente discutidos, estudiados y recientemente revisados por Bianchi (2005). Sin embargo, en Uruguay los antecedentes son escasos, particularmente

Aceptado: 20.12.2005

${ }^{1}$ Ruta 3 km 363.500. Paysandú. 60000. URUGUAY. Fax : (00) 598

72 27950. E-mail: tano@fagro.edu.uy en calidad de la carne (INAC, INIA, CSU, 2003, Bianchi y Garibotto 2004).

El objetivo del presente trabajo es evaluar el efecto del peso al sacrificio (corderos livianos: $22,3 \pm 2,8 \mathrm{~kg}$ y $60 \pm 2,4$ días de edad y corderos pesados: $43,1 \pm 6,3 \mathrm{~kg}$ de peso vivo y $169 \pm 8,9$ días de edad) sobre la calidad de la canal y de la carne de corderos (hembras, machos criptórquidos y castrados) Corriedale puros y cruza Hampshire Down x Corriedale y Southdown x Corriedale.

\section{MATERIAL Y METODOS}

1. LOCALIZACION Y PERIODO EXPERIMENTAL. El trabajo se desarrolló en las instalaciones de la Estación Experimental "Dr. Mario A. Cassinoni" (EEMAC), de la Facultad de Agronomía, Paysandú, Uruguay $\left(32,5^{\circ}\right.$ de latitud sur y $58,0^{\circ}$ de longitud oeste) durante el período 27/8/2002-17/2/2003.

2. ANIMALES, MANEJO Y TRATAMIENTOS. Se utilizaron 66 corderos que fueron estratificados por sexo (24 hembras, 23 machos criptórquidos y 19 castrados) y tipo genético (21 Corriedale puros: C, 23 cruza Hampshire Down x Corriedale: HD x C y 22 Southdown x Corriedale: SD x C), 
y asignados a dos tratamientos de peso y edad para ser sacrificados como: corderos "livianos" (n: 45; 22,3 $\pm 2,8$ $\mathrm{kg}$ de peso vivo y $60 \pm 2,4$ días de edad) o corderos "pesados" (n: $21 ; 43,1 \pm 6,3 \mathrm{~kg}$ de peso vivo y $169 \pm 8,9$ días de edad). Los animales provenían de 13 carneros: 5 Corriedale, 4 Hampshire Down y 4 Southdown. Desde el parto a fines de agosto y hasta el embarque de los corderos (28/10/2002 en caso de corderos "livianos" y $17 / 2 / 2003$ en caso de corderos "pesados"), todos los animales se mantuvieron sobre pasturas sembradas anuales y plurianuales. El destete se realizó el 7/1/2003 con un peso vivo de $35,8 \pm 6,14 \mathrm{~kg}$.

\section{METODOLOGIA}

3.1. Controles en la canal. Los sacrificios de cada tipo comercial evaluado se realizaron en una única ocasión y cuando los animales alcanzaron el peso vivo y el grado de terminación requerido, en el Frigorífico Casa Blanca de Paysandú (distante de la EEMAC $23 \mathrm{~km}$ ). Una vez en la planta, y tras 15 horas de espera con acceso al agua, se procedió al sacrificio de los animales siguiendo las pautas estándar para la obtención de cortes de exportación.

En la canal fría $\left(24 \mathrm{~h} \mathrm{a} 4^{\circ} \mathrm{C}\right)$ de corderos "livianos" $(11,2 \pm 1,83 \mathrm{~kg})$ y "pesados" $(23,2 \pm 5,3 \mathrm{~kg})$ se determinó la conformación en forma objetiva recurriendo a las medidas morfológicas de longitud total de la canal; longitud y anchura de pierna, descritas por Fisher y de Boer (1994) y Ruiz de Huidobro y col (2000). Con esta información se calculó el índice de compacidad de la canal (ICC) como el peso canal fría dividido por la longitud de la canal y el índice de compacidad de la pierna (ICP), como la anchura de la pierna dividido por la longitud de esta. El grado de engrasamiento se determinó a través de la profundidad de los tejidos sobre la $12^{\mathrm{a}}$ costilla a $11 \mathrm{~cm}$ de la línea media: punto GR (Kirton y Jonson 1979). Posteriormente se realizó el despiece comercial de la canal en carne de categoría I, II y III (Vergara y Gallego 2000). Las piezas comerciales se pesaron y se agruparon en las siguientes categorías: Categoría I: pierna + costillar + badal; Categoría II: espalda o paletilla, y Categoría III: bajos (falda o pecho) + cuello.

En la Figura 1, se ilustra el despiece realizado.

Posteriormente, se procedió a retirar de la media canal izquierda la espalda (Colomer-Rocher y col 1988), la que después de pesada, se envasó al vacío y se congeló a $-35^{\circ} \mathrm{C}$. Posteriormente y tras una descongelación lenta a temperatura ambiente durante $24 \mathrm{~h}$, se procedió a la disección de la espalda: peso total de la espalda, grasa subcutánea, grasa intermuscular, grasa preescapular, grasa total, músculo, hueso (hueso y cartílago) y desechos (fascias, ligamentos y vasos sanguíneos). Con esta información se calculó la relación músculo/grasa y músculo/ hueso.

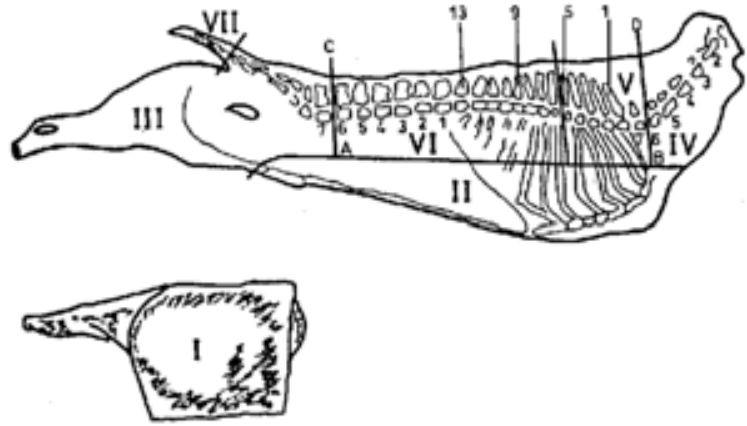

Orden de obtención de las piezas:

I. Espalda

II. Bajos

III. Pierna

IV. Cuello

V. Badal

VI. Costillar

VII. Cola

Figura 1. Diagrama de cortes de la canal. Diagram of the carcass cuts.

3.2. Controles en la carne. Sobre muestras del músculo Longissimus dorsi y tras 24 h de maduración, se midió el pH (Garrido y Bañón 2000), la capacidad de retención de agua (CRA, Plá 2000), pigmentos hemínicos (análisis químico por espectrofotometría, con lectura a $640 \mathrm{~nm}$; Albertí, 2000) y textura con la célula de cizalla de Warner-Bratzler (Beltrán y Roncales 2000). El filete del músculo Longissimus dorsi, destinado al análisis de textura que había sido adecuadamente condicionado y congelado tras 24 h de maduración, se pesó tras la descongelación y tras la cocción a baño María hasta alcanzar una temperatura interna de $70^{\circ} \mathrm{C}$. La diferencia de ambos pesos, dividido por el peso tras la descongelación, se utilizó para calcular las pérdidas por cocción (PPC).

Para el análisis sensorial, filetes del músculo Longissimus dorsi de estos animales fueron descongelados en agua corriente hasta alcanzar los $16,2 \pm 2,03^{\circ} \mathrm{C}$ de temperatura interna y luego se procedió de acuerdo a la metodología descrita por Guerrero (2000). Se utilizó una muestra de 49 personas (11 mujeres y 38 hombres) con una edad media de $42 \pm 11,3$ años y mayoritariamente sin hábitos de consumo de carne ovina (76\% de los consumidores consumían carne ovina una o menos veces al mes). Los consumidores trabajaron en el Laboratorio de Calidad de Carne de la EEMAC en 5 sesiones de $1 \mathrm{~h}$ de duración cada una, evaluando un total de 21 platos de 2-3 muestras cada uno (diseño en bloques incompletos y desbalanceados). Para el test se utilizó una planilla con escala discontinua estructurada y tres atributos: grado de terneza, calidad de sabor y aceptabilidad con valores de 1 a 10, incrementando el puntaje conforme la intensidad del atributo mejoraba. 
4. ANALISIS ESTADISTICO. El efecto del peso al sacrificio sobre todas las variables de calidad de canal, $\mathrm{pH}, \mathrm{CRA}$, pigmentos hemínicos, PPC y textura, se estudió mediante análisis de varianza considerando un modelo fijo y utilizando el método de mínimos cuadrados, provisto por el procedimiento GLM del paquete estadístico SAS versión 8,0 (SAS, Institute Inc 1998), considerando la suma de cuadrados tipo III. Para el análisis sensorial de las variables solicitadas en la hoja de escala estructurada (terneza, calidad de sabor y aceptabilidad de la muestra) se utilizó un modelo mixto que incluyó como efectos fijos: tipo genético (3 niveles), sexo del cordero (3 niveles), peso al sacrificio (2 niveles) y las interacciones entre los efectos, considerando los efectos consumidor, plato anidado a consumidor y orden de la muestra como aleatorios. Se utilizó el procedimiento MIXED del paquete estadístico SAS versión 8,0 (SAS, Institute, Inc 1998).

\section{RESULTADOS Y DISCUSION}

En el cuadro 1 se presenta el efecto del peso al sacrificio sobre la conformación, engrasamiento y composición tisular de la canal.

El peso al sacrificio afectó $(\mathrm{P} \leq 0,05)$ todas las características de la canal evaluadas, los corderos pesados presentaran mejor conformación $(0,320$ vs 0,190 kg/cm, 1,09

Cuadro 1. Efecto del peso al sacrificio sobre algunas características de la canal de corderos machos y hembras Corriedale puros y cruza Hampshire Down x Corriedale y Southdown x Corriedale.

Effect of live weight at slaugther on some carcas characteristics of male and female pure-bred Corriedale and crossbred lambs.

\begin{tabular}{lcc}
\hline \multicolumn{1}{c}{ Característica de la canal } & $\begin{array}{c}\text { Cordero } \\
\text { liviano }\end{array}$ & $\begin{array}{c}\text { Cordero } \\
\text { pesado }\end{array}$ \\
\hline Conformación y engrasamiento & $(\mathrm{n}=45)$ & $(\mathrm{n}=21)$ \\
Largo canal (cm) & $59,8 \pm 0,50 \mathrm{~b}$ & $71,0 \pm 0,74 \mathrm{a}$ \\
Ancho tórax (cm) & $17,1 \pm 0,25 \mathrm{~b}$ & $23,4 \pm 0,37 \mathrm{a}$ \\
Profundidad tórax (cm) & $20,9 \pm 0,18 \mathrm{~b}$ & $27,0 \pm 0,27 \mathrm{a}$ \\
Largo pierna (cm) & $17,6 \pm 0,16 \mathrm{~b}$ & $21,0 \pm 0,23 \mathrm{a}$ \\
Perímetro de pierna (cm) & $54,4 \pm 0,39 \mathrm{~b}$ & $62,5 \pm 0,59 \mathrm{a}$ \\
Compacidad de pierna & $1,07 \pm 0,01 \mathrm{~b}$ & $1,09 \pm 0,01 \mathrm{a}$ \\
Compacidad de canal (kg/cm) & $0,19 \pm 0,05 \mathrm{~b}$ & $0,32 \pm 0,01 \mathrm{a}$ \\
Composición tisular de la espalda & & \\
Carne (\%) & $49,4 \pm 0,84 \mathrm{~b}$ & $56,5 \pm 1,21 \mathrm{a}$ \\
Hueso (\%) & $27,2 \pm 0,49 \mathrm{~b}$ & $19,9 \pm 0,49 \mathrm{~b}$ \\
Grasa Total (\%) & $23,4 \pm 0,74 \mathrm{a}$ & $23,6 \pm 1,06 \mathrm{a}$ \\
Grasa subcutánea/grasa total $(\%)$ & $48,2 \pm 2,12 \mathrm{a}$ & $41,0 \pm 3,06 \mathrm{~b}$ \\
Grasa intermuscular/grasa total (\%) & $33,6 \pm 0,05 \mathrm{~b}$ & $39,1 \pm 3,50 \mathrm{a}$ \\
Grasa pre escapular/grasa total (\%) & $18,2 \pm 0,88 \mathrm{a}$ & $19,9 \pm 1,27 \mathrm{a}$ \\
Carne /grasa & $2,1 \pm 0,11 \mathrm{~b}$ & $2,4 \pm 0,15 \mathrm{a}$ \\
Carne/hueso & $1,8 \pm 0,06 \mathrm{~b}$ & $2,8 \pm 0,09 \mathrm{a}$ \\
\hline
\end{tabular}

(a, b): Valores seguidos por diferente letra difieren en $\mathrm{P} \leq 0,05$. vs. $1,07,55,7$ vs. 51,9 compacidad de la canal y de la pierna de corderos pesados y livianos, respectivamente), superior grado de engrasamiento $(15,7 \mathrm{vs} .7,3 \mathrm{~mm}$ punto GR de canales de corderos pesados y livianos, respectivamente) y composición tisular más favorable (2,8 vs. 1,8 carne/ hueso, 2,4 vs. 2,1 carne/grasa, corderos pesados y livianos, respectivamente).

En el cuadro 2 se presenta el efecto del peso al sacrificio sobre la composición regional de la canal.

Las canales de corderos livianos presentaron una mejor composición regional que las de los corderos pesados, particularmente en aquellas categorías que contemplan los cortes de mayor valor comercial. La mayor contribución relativa de pierna en los corderos livianos frente a los pesados es compatible con el desarrollo más temprano de esta pieza en particular; explicando que el resultado se invierta cuando se considera una pieza de desarrollo más tardío como es el caso del costillar. Asimismo, la mayor contribución relativa de la espalda en los corderos livianos es compatible con la conformación registrada en estas canales y coincide con lo reportado por Ruiz de Huidobro y col (2000). Estos autores señalan que las canales largas y mal conformadas tienen mayor porcentaje de piezas magras (como, por ejemplo, la espalda), mientras que las canales compactas tienen mayor proporción de piezas grasas (como, por ejemplo, el costillar).

En el cuadro 3 se presenta el efecto del peso al sacrificio sobre la calidad de la carne.

De las características de calidad instrumental de la carne sólo el pH resultó modificado por efecto del peso al sacrificio, presentando los corderos livianos lecturas altas y superiores a lo deseable ( $\leq 5,8$; Sañudo 1992). Es

Cuadro 2. Efecto del peso al sacrificio sobre la composición regional de la canal de corderos machos y hembras Corriedale puros y cruza Hampshire Down x Corriedale y Southdown x Corriedale.

Effect of live weight at slaughter on carcass regional composition of male and female pure-bred Corriedale and crossbred lambs.

\begin{tabular}{lcc}
\hline $\begin{array}{l}\text { Clasificación de } \\
\text { la canal y contribución } \\
\text { relativa de las piezas }\end{array}$ & $\begin{array}{c}\text { Cordero } \\
\text { liviano } \\
(\mathrm{n}=45)\end{array}$ & $\begin{array}{c}\text { Cordero } \\
\text { pesado } \\
(\mathrm{n}=21)\end{array}$ \\
\hline Categoría I (\%) & $58,5 \pm 0,46 \mathrm{a}$ & $52,5 \pm 0,71 \mathrm{~b}$ \\
Pierna & $57,9 \pm 0,46 \mathrm{a}$ & $55,4 \pm 0,70 \mathrm{~b}$ \\
Badal & $10,9 \pm 0,34 \mathrm{a}$ & $10,0 \pm 0,52 \mathrm{a}$ \\
Costillar & $31,2 \pm 0,44 \mathrm{~b}$ & $34,6 \pm 0,68 \mathrm{a}$ \\
Categoría II (\%) & $18,8 \pm 0,16 \mathrm{a}$ & $17,9 \pm 0,25 \mathrm{~b}$ \\
Espalda o paletilla & $100 \mathrm{a}$ & $100 \mathrm{a}$ \\
Categoría III $(\%)$ & $19,8 \pm 0,25 \mathrm{~b}$ & $25,4 \pm 0,38 \mathrm{a}$ \\
Bajos $(\%)$ & $66,5 \pm 0,73 \mathrm{~b}$ & $75,3 \pm 1,12 \mathrm{a}$ \\
Cuello (\%) & $33,5 \pm 0,73 \mathrm{a}$ & $24,7 \pm 1,12 \mathrm{~b}$ \\
\hline
\end{tabular}

(a, b): Valores seguidos por diferente letra difieren en $\mathrm{P} \leq 0,05$. 
Cuadro 3. Efecto del peso al sacrificio sobre características de la carne de corderos machos y hembras Corriedale puros y cruza Hampshire Down x Corriedale y Southdown x Corriedale. Effect of live weight at slaughter on meat characteristics of male and female pure-bred Corriedale and crossbred lambs.

\begin{tabular}{lcc}
\hline Calidad de carne & $\begin{array}{c}\text { Cordero } \\
\text { liviano }(\mathrm{n}=45)\end{array}$ & $\begin{array}{c}\text { Cordero } \\
\text { pesado }(\mathrm{n}=21)\end{array}$ \\
\hline $\mathrm{pH}$ & $5,9 \pm 0,01 \mathrm{a}$ & $5,6 \pm 0,01 \mathrm{~b}$ \\
CRA (\% de jugo liberado) & $16,4 \pm 0,71 \mathrm{a}$ & $14,4 \pm 1,09 \mathrm{a}$ \\
PPC (\%) & $19,0 \pm 0,69 \mathrm{a}$ & $15,8 \pm 1,07 \mathrm{~b}$ \\
Color (mg mioglobina/g & $3,02 \pm 0,46 \mathrm{a}$ & $3,73 \pm 0,68 \mathrm{a}$ \\
Músculo) & $4,7 \pm 0,25 \mathrm{a}$ & $4,1 \pm 0,38 \mathrm{a}$ \\
Textura (kg) & & \\
\hline
\end{tabular}

(a, b): Valores seguidos por diferente letra difieren en $\mathrm{P} \leq 0,05$. PPC: pérdida por cocción; CRA: capacoaca retención de agua.

probable que a ello haya contribuido más el estrés de estos corderos, ocasionado por la separación abrupta de sus madres al momento del sacrificio, que el peso en sí mismo, en virtud de la incidencia que distintos factores tienen sobre la velocidad de caída del pH, como sobre su valor final (Bianchi 2005).

El hecho de que el peso al sacrificio prácticamente no afectara las características de la carne coincide con el postulado que sostiene que las características intrínsecas o productivas, en general, influyen mayoritariamente sobre la calidad de la canal, resultando bastante menor su importancia relativa sobre los atributos de calidad de carne, sobre todo, en corderos (Sañudo y col 1998), donde, además, los corderos sacrificados más tarde eran igualmente muy jóvenes.
En el cuadro 4 se presenta el efecto del sexo, del tipo genético y del peso al sacrificio sobre los atributos de terneza, calidad de sabor y aceptabilidad de la carne de cordero.

De las interacciones analizadas, la única que resultó significativa $(\mathrm{P}=0,02)$ fue tipo genético $\mathrm{x}$ peso al sacrificio: los animales cruza, pero no los puros, mejoraron la terneza de su carne al pasar de cordero liviano a pesado. El sexo del cordero y el tipo genético afectaron sólo la variable terneza, resultando más tierna la carne de corderas hembras o machos castrados versus corderos criptórquidos, particularmente si eran cruza. Bianchi (2005) también reporta que la carne de los corderos cruza resultaba más tierna sensorialmente que la de corderos Corriedale puros.

El peso al sacrificio afectó todas las características organolépticas, recibiendo la carne de corderos pesados mejor notación por los consumidores. El mayor engrasamiento de estos corderos asociado a una mejora en la jugosidad y a una menor susceptibilidad al acortamiento por frío de estas canales (Sañudo 1992, Martínez-Cerezo 2005), probablemente explique la mejor apreciación global que los consumidores le asignaron a la carne de los corderos pesados.

La mayor sensibilidad del análisis sensorial frente al instrumental (Martínez-Cerezo 2005) explicaría que la tendencia ya manifiesta en la textura instrumental (menor fuerza de corte en la carne proveniente de los corderos más pesados, cuadro 3) resulte sensorialmente significativa, obteniéndose carne más tierna conforme el peso al sacrificio aumentó (cuadro 4).

Los resultados del presente trabajo sugieren que la decisión de qué tipo de cordero producir afecta mayor-

Cuadro 4. Evaluación de consumidores sobre atributos de la carne de corderos machos y hembras, livianos y pesados, Corriedale puros y cruza.

Consumer sensory evaluation of meat from male and female light and heavy pure-bred Corriedale and crossbred lambs.

\begin{tabular}{lccc}
\hline & $\begin{array}{c}\text { Terneza } \\
(1-10)^{1}\end{array}$ & $\begin{array}{c}\text { Calidad de sabor } \\
(1-10)^{1}\end{array}$ & $\begin{array}{c}\text { Aceptabilidad } \\
(1-10)^{1}\end{array}$ \\
\hline SEXO & + & $\mathrm{ns}$ & $\mathrm{ns}$ \\
Cordera hembra $(\mathrm{n}=24)$ & $6,9 \pm 0,24$ & $6,8 \pm 0,22$ & $6,8 \pm 0,23$ \\
Cordero criptórquido $(\mathrm{n}=23)$ & $6,3 \pm 0,26$ & $7,0 \pm 0,23$ & $6,9 \pm 0,24$ \\
Cordero castrado $(\mathrm{n}=19)$ & $6,9 \pm 0,23$ & $7,1 \pm 0,21$ & $7,1 \pm 0,22$ \\
TIPO GENETICO & $*$ & $\mathrm{~ns}$ & $\mathrm{~ns}$ \\
C (n= 21) & $6,2 \pm 0,25 \mathrm{~b}$ & $6,8 \pm 0,23$ & $6,7 \pm 0,24$ \\
HD x C (n=23) & $6,7 \pm 0,23 \mathrm{ab}$ & $7,0 \pm 0,22$ & $6,9 \pm 0,23$ \\
SD x C (n=22) & $7,2 \pm 0,28 \mathrm{a}$ & $7,2 \pm 0,25$ & $7,2 \pm 0,27$ \\
PESO AL SACRIFICIO & $* *$ & $* * *$ & $* * *$ \\
Corderos livianos $(\mathrm{n}=45)$ & $6,3 \pm 0,28 \mathrm{~b}$ & $6,6 \pm 0,20 \mathrm{~b}$ & $6,5 \pm 0,21 \mathrm{~b}$ \\
Corderos pesados $(\mathrm{n}=21)$ & $7,1 \pm 0,25 \mathrm{a}$ & $7,3 \pm 0,23 \mathrm{a}$ & $7,4 \pm 0,24 \mathrm{a}$ \\
\hline
\end{tabular}

ns: $\mathrm{P}>0,05 ;(+): \mathrm{P} \leq 0,05 ;(*): \mathrm{P} \leq 0,01 ;(* *): \mathrm{P} \leq 0,005 ;(* * *): \mathrm{P} \leq 0,0001 ;(\mathrm{a}, \mathrm{b})$ : Valores seguidos por diferente letra en la misma columna difieren en $\mathrm{P} \leq 0,01$.

(1): Escala de puntaje. 
mente las características de calidad de la canal (en particular la conformación y la distribución de los diferentes tipos de grasa en relación a la grasa total), frente a las de la carne, a pesar de que el consumidor uruguayo prefiere la carne de corderos pesados, particularmente si éstos son cruza.

\section{RESUMEN}

Se estudió el efecto del peso al sacrificio (45 corderos livianos: $22,3 \pm 2,8 \mathrm{~kg}$ y $60 \pm 2,4$ días de edad y 21 corderos pesados: $43,1 \pm 6,3 \mathrm{~kg}$ de peso vivo y $169 \pm 8,9$ días de edad) sobre la calidad de la canal y de la carne de 24 hembras, 23 machos criptórquidos y 19 corderos castrados Corriedale puros $(\mathrm{n}=21)$, Hampshire Down $x$ Corriedale $(\mathrm{n}=23)$ y Southdown $x$ Corriedale $(n=22)$. El peso al sacrificio afectó $(P \leq 0,05)$ todas las características de la canal evaluadas. Los corderos pesados presentaran mejor conformación (0,320 vs. $0,190 \mathrm{~kg} / \mathrm{cm}, 1,09$ vs. $1,07,55,7$ vs. 51,9 compacidad de la canal y de la pierna de corderos pesados y livianos, respectivamente), superior grado de engrasamiento $(15,7$ vs. $7,3 \mathrm{~mm}$ punto GR de canales de corderos pesados y livianos, respectivamente) y composición tisular más favorable (2,8 vs. 1,8 carne/ hueso, 2,4 vs. 2,1 carne/ grasa, corderos pesados y livianos, respectivamente). Sin embargo, las canales de corderos livianos presentaron un mejor rendimiento comercial de cortes nobles que las de los corderos pesados. De las características de calidad instrumental de la carne sólo el $\mathrm{pH}$ resultó modificado por efecto del peso al sacrificio. Sensorialmente el sexo del cordero y el tipo genético afectaron sólo la variable terneza, resultando más tierna la carne de corderas hembras o machos castrados versus corderos criptórquidos, particularmente si eran cruza. El peso al sacrificio afectó todas las características organolépticas, recibiendo la carne de corderos pesados mejor puntaje en terneza, calidad de sabor y aceptabilidad por los consumidores.

\section{REFERENCIAS}

Albertí P. 2000. Medición del color. En: Metodología para el estudio de la calidad de la canal y de la carne en rumiantes. Ministerio de Ciencia y Tecnología-INIA. Madrid, España, pp. 159-166.

Beltrán JA, P Roncalés. 2000. Determinación de la textura. En: Metodología para el estudio de la calidad de la canal $y$ de la carne en rumiantes. Ministerio de Ciencia y Tecnología-INIA. Madrid, España, pp. 169-172.

Bianchi G, G Garibotto. 2004. Identificación y cuantificación de factores que afectan la calidad de carne ovina. En: $1^{e r}$ Seminario Técnico. Calidad de Carne Ovina y Vacuna: Impacto de decisiones tomadas en distintos segmentos de la cadena. (ed.) Bianchi, G. y Garibotto, G. Facultad de Agronomía, EEMAC. FRICASA. Paysandú, Uruguay. Pp 52.

Bianchi G. 2005. Características productivas, tipificación de la canal y calidad de carne a lo largo de la maduración de corderos pesados Corriedale puros y cruzados en sistemas extensivos. Tesis Doctoral. Universidad de Zaragoza, Facultad de Veterinaria, Zaragoza, España. Pp 102.
Colomer-Rocher F, R Delfa, I Sierra. 1988. Métodos normalizados para el estudio de los caracteres cuantitativos y cualitativos de las canales ovinas producidas en el área mediterránea, según los sistemas de producción. En: Métodos normalizados para el estudio de los caracteres cuantitativos y cualitativos de las canales caprinas y ovinas. Cuadernos INIA 17, 19-41.

Fisher A, H De Boer. 1994. The EAAP standard meted sheep carcass assessment. Carcass measurements and dissection procedures. Livest Prod Sci 38, 149-159.

Garrido MD, S Bañón. 2000. Medidas del pH. En: Metodología para el estudio de la calidad de la canal y de la carne en rumiantes. Ministerio de Ciencia y Tecnología-INIA. Madrid, España. Pp 147-155.

Guerrero, L. 2000. Determinación sensorial de la calidad de la carne. En: Metodología para el estudio de la calidad de la canal y de la carne en rumiantes. Ministerio de Ciencia y Tecnología-INIA. Madrid, España. Pp 207-220.

Hudson IW, HA Gimp, PG Woolfolk, JD Kemp, CM Reese. 1968. Effect of induced cryptorchidism at different weights on performance and carcass traits of lambs. J Anim Sci 27, 45-7.

INIA, INAC, CSU. 2003. Auditoría de Calidad de Carne Ovina. "Un compromiso de mejora continua de la calidad de la carne ovina de Uruguay". Pp 28.

Kirton A H, DL Johnson. 1979. Interrelationships between GR and other lamb carcass fatness measurements. Proceedings of the New Zealand Society of Animal Production 39, 194201.

Martínez-Cerezo, M.S. 2005. Calidad instrumental y sensorial de la carne ovina. Influencia de la raza, del peso al sacrificio y del tiempo de maduración. Tesis Doctoral. Universidad de Zaragoza, Facultad de Veterinaria, Zaragoza, España. Pp 290.

Plá M. 2000. Medida de la capacidad de retención de agua. En: Metodología para el estudio de la calidad de la canal $y$ de la carne en rumiantes. Ministerio de Ciencia y Tecnología-INIA. Madrid, España. Pp 175-179.

Ruiz de Huidobro F, V Cañeque, E Onega, S Velasco. 2000. Morfología de la canal ovina. En: Metodología para el estudio de la calidad de la canal y de la carne en rumiantes. Ministerio de Ciencia y Tecnología-INIA. Madrid, España. $P p$ 83-102.

Salgado C. 2003. El mercado mundial de carne ovina. En: $12^{\circ}$ Congreso Mundial de la Raza Corriedale. 1-10/9/2003. Montevideo, Uruguay. Conferencia (CD-ROOM) y 91-96.

Sañudo C. 1992. La calidad organoléptica de la carne con especial referencia a la especie ovina. Factores que la determinan, métodos de medida y causas de variación. Pp 117.

Sañudo C, A Sanchez, A Alfonso. 1998. Small ruminant production systems and factors affecting lamb meat quality. Meat Sci 49, S29-S64.

SAS. Institute Inc., SAS/STAT. User's Guide, versión 8.0. Carey, N.C. 1998.

Vergara H, L Gallego. 2000. Composición de la canal ovina. En: Metodología para el estudio de la calidad de la canal y de la carne en rumiantes. Ministerio de Ciencia y Tecnología-INIA. Madrid, España. Pp 127-136. 